\title{
Towards Finding Global Representations of the Efficient Set in Multiple Objective Mathematical Programming
}

\author{
Harold P. Benson \\ University of Florida, College of Business Administration. \\ Gainesville, Florida 32611, USA \\ Serpil Sayin \\ Bilkent University, Faculty of Business Administration, 06533 Bilkent, Ankara. Turkey
}

\begin{abstract}
We propose and justify the proposition that finding truly global representations of the efficient sets of multiple objective mathematical programs is a worthy goal. We summarize the essential elements of a general global shooting procedure that seeks such representations. This procedure illustrates the potential benefits to be gained from procedures for globally representing efficient sets in multiple objective mathematical programming. C. 1997 John Wiley \& Sons. Inc.
\end{abstract}

\section{INTRODUCTION}

The multiple objective mathematical programming problem (MMP) may be written

$$
\text { (MMP) "maximize" } f(z), \quad \text { subject to } z \in Z,
$$

where $f=\left[f_{1}, f_{2}, \ldots f_{l}\right]$ is a vector-valued function involving $p \geq 2$ real-valued objective functions $f_{l}, j=1,2, \ldots, p$, defined on a nonempty feasible region $Z \subseteq R^{h}$. Problem (MMP) has received a great deal of attention during the past 20 years, because numerous real-world applications involve more than one objective function. Examples of applications of problem (MMP) can be found in a wide variety of fields, including production planning (Gravel et al. [27]), scheduling (Bagchi [4], Prabuddha, Ghosh, and Wells [40]), environmental policy (Leschine, Wallenius, and Verdini [34]), nutrition planning (Benson and Morin [12]), and numerous others (cf.. e.g., Goicoechea, Hansen, and Duckstein [26], Stadler [44], Steuer [47], White [50], Zeleny [54], and references therein). As explained in various texts and review articles (see, e.g.. Cohon [15], Keeney and Raiffa [32], Rosenthal [41], Yu [51], and Zeleny [54]), problem (MMP) is typically solved by a decision maker (DM) who, with the aid of an analyst, searches in $Z$ for a most preferred solution. Whenever the DM feels that "more is better" for each objective function $f_{i}$ in problem (MMP), these searches can be confined to the subset of efficient solutions. where an efficient solution is defined as follows.

DEFINITION 1: A point $z^{0}$ is said to be an efficient solution for problem (MMP) when $z^{10} \in Z$ and there exists no point $z \in Z$ such that $f(z) \geq f\left(z^{0}\right)$ and $f(z) \neq f\left(z^{0}\right)$.

We will use the notation $Z_{E}$ to represent the set of efficient solutions for problem 
(MMP). Solution procedures for problem (MMP) can be classified into three categories of approaches according to the time at which the DM must express his preferences among alternative solutions (cf. Dyer et al. [20], Evans [23], Hwang and Masud [30]). In particular, each solution procedure either requests preference information from the DM prior to, during, or after some type of optimization phase.

Unfortunately, none of the procedures for implementing these approaches is wholly adequate. Each procedure either makes overly restrictive assumptions, demands information that is beyond the ability of the DM to provide, calls for excessive computation, or fails to adequately represent the efficient set.

The purpose of this article is twofold. The first purpose is to suggest to researchers that in order to help solve problem (MMP), a worthy goal is to attempt to derive truly global representations of the efficient set. The second purpose is to illustrate the potential benefits of this goal by conveying the essence of an approach that we have recently formulated that seeks global representations of $Z_{t}$ for many cases of the problem. The approach is imbedded in a procedure that we call the global shooting procedure. The essence of the approach is to transform the problem to a lower-dimensional space wherein a simplex containing the feasible region of the problem is constructed. Global representations of the efficient set of the original problem (MMP) are then obtained by shooting toward a certain facet $S^{0}$ of this simplex, starting from a special point that does not lie on $S^{0}$.

The content of this article is as follows. In Section 2 we first give a brief critique of the strengths and weaknesses of current approaches for solving problem (MMP). We then propose and justify the proposition that finding global representations of the efficient set of problem (MMP) would be a valuable goal. In Section 3, we summarize the derivation and steps of the global shooting procedure, and we illustrate its use on a simple example. To demonstrate some of the possible implementations of the global shooting procedure, Section 4 reports some experimental results obtained by using the procedure four times to generate four different global representations of the efficient set of a sample problem. Conclusions and directions for further research are given in the last section. For brevity, proofs of theorems are omitted. The interested reader is referred to Sayin [42] for proofs and further details.

\section{CURRENT APPROACHES AND A PROPOSITION}

As a preliminary to proposing an appropriate strategy for solving problem (MMP), we first present a very brief critical review of the three current approaches for solving the problem. Readers desiring a more detailed analysis are referred to $[15,20,23,26,30,32,33$, $41-43,47,51,54]$ and to references therein.

The a priori procedures for solving problem (MMP) seek first to derive a value function $v$ that represents the preferences of the DM. Subsequently, a most preferred solution $z^{*}$ is found by maximizing $v[f(z)]$ over all points $z$ in $Z$ and choosing any optimal solution thereby obtained as $z^{*}$. The attraction of the a priori methods is that after a value function $v$ has been found, the optimization phase is often accomplished relatively easily.

A priori procedures, however, are often not applicable to solving problem (MMP). This is because, to use them, the DM's preference structure is required to satisfy conditions that tend to be quite restrictive $[32,33,53]$. Even when an a priori method can be used, the value function $v$ that it gives is frequently an inaccurate reflection of the DM's true preference structure. This is because to derive $v$, the DM is often asked to give preference judgments that are very difficult to determine. Furthermore, a priori methods provide only one 
solution, and this solution need not belong to $Z_{1}$. This is undesirable in situations where the more is better assumption holds and many solutions may be consistent with the DM's preferences.

A second set of procedures for solving problem (MMP), called interactive methods, consist of DM-machine interactions that generate a discrete sample of points in $Z$ or in $Z_{\text {i }}$. $[1,43,47]$. During each iteration of a typical interactive algorithm, a computer program first finds a point in $Z$ or in $Z_{k}$ by solving an appropriate single-objective optimization problem. Next, the DM is asked to assess his or her relative preference for this solution. Based upon this information, the single-objective optimization problem is modified. and another iteration begins. These DM-machine interactions are repeated until the DM indicates that the current solution is a most preferred solution to problem (MMP). A key attraction of interactive methods is that the DM can typically more easily and accurately supply the required preference information than in a priori methods. Also, interactive algorithms allow the DM to explore portions of $Z$ or of $Z_{l}$, and they are computationally tractable.

Unfortunately, however, in practice the portion of $Z$ or of $Z_{l}$ explored by an interactive algorithm is invariably quite small. This is mainly due to the limited information processing ability and stamina of the DM. Because of this, the DM usually terminates the search after only a handful of points in $Z$ or in $Z_{I}$ : have been found $[11,47]$. In addition, some interactive methods have been criticized for failing to generate points in $Z_{l}$, and others use arbitrary, unverified search paradigms.

The third category of solution approaches for problem (MMP) consists of procedures that do not call upon the DM to express preferences among alternative solutions until after the optimization phase has been accomplished. In this approach, often called the vector maximization approach ( $\mathrm{cf}$. Geoffrion [25]), the goal is to generate either all of $Z_{l}$, or a substantial portion of $Z_{1}$, so that the DM can choose a most preferred solution from the generated set. The main strengths of the vector maximization approach are that it provides a range of solutions from $Z_{1}$ for the DM to study and choose from and that the only assumption that it needs to apply, namely that more is better, is almost always satisfied.

The vector maximization approach seems to offer promise, but implementations to date are not yet adequate. There are several reasons for this.

The main disappointment of the current implementations of the vector maximization approach is that they do not attempt to generate truly global. representative subsets of $Z_{1}$. Instead, the procedures in this class either attempt to generate all of $Z_{\ell}$ or arbitrary subsets of $Z_{l}$ that do not necessarily globally represent $Z_{l}$.

Because $Z_{l}$ is generally a large and complicated nonconvex set, generating it in its entirety is only possible in certain special cases (cf., e.g., Benson [7], Isermann [31], Ecker. Hegner, and Kouada [21], Bitran [13], Villarreal and Karwan [49]). Even in these special cases, the computational effort required to generate all of $Z_{1}$ becomes rapidly un manageable and seems to grow exponentially with problem size (cf., e.g., Evans and Steuer [24], Marcotte and Soland [36] and Steuer [47]). Furthermore, the sheer size of the efficient set becomes so great that it becomes difficult to present and can overwhelm the DM to the extent that he or she cannot choose a most preferred solution (cf. Steuer [45]).

In some cases of problem (MMP), vector maximization procedures have been used to generate certain subsets of $Z_{l}$. For instance, several methods have been proposed for generating the set of efficient extreme points and directions for a multiple objective linear program (see, e.g., Ecker and Kouada [22], Yu and Zeleny [52], and Evans and Steuer [24]). It was soon discovered, however, that arbitrarily chosen subsets of $Z_{l}$ such as these 
do not necessarily provide adequate global representations of $Z_{l}$. Furthermore, like $Z_{l}$ : itself, it was found that such subsets could also be too large to generate and could overwhelm the DM [17, 18, 37, 46-48].

It is our contention that if the goal of the vector maximization approach were redirected to explicitly generate a truly globally representative but manageable subset of $Z_{l}$, then the vector maximization approach could potentially alleviate many of the weaknesses of the present three approaches for solving problem (MMP). Indeed, a few researchers have occasionally alluded to this goal, but it has never been explicitly discussed or pursued (cf.. e.g., Armann [3] and Steuer [47]). Instead, most researchers seem to have continued to pursue algorithms that use local search ideas from traditional linear and nonlinear programming. They seem to have done so in spite of the fact that these methods were never intended to give a global or statistically representative sample of a large, complicated nonconvex set such as $Z_{I:}$.

In particular, we propose that the goal of the first step of the vector maximization approach ought to be to generate a globally representative sample of points from $Z_{I:}$ of as manageable a size as possible. Given such a set, if it were of a reasonable size, the DM could then choose a most preferred solution from it. Recent advances in the field of global optimization could potentially contribute significantly toward the achievement of this goal (cf. Horst and Pardalos [28], Horst and Tuy [29]). ldeally, the sample of points generated would provide a valid global representation of $Z_{K}$, perhaps in a statistical sense, without being redundant or overwhelming the DM.

In the next section we present a method called the global shooting procedure to illustrate some of the potential benefits of generating global representations of $Z_{L}$. The reader should bear in mind that the global shooting procedure is presented as simply one concrete example of how one might begin to attempt to achieve what we feel is a worthy goal of generating manageable global representations of efficient sets. It is our hope that other attempts of this sort will follow.

\section{A GLOBAL SHOOTING PROCEDURE}

The global shooting procedure works in the outcome space $\mathbb{R}^{\prime}$ of problem (MMP) rather than in the decision space $\mathbb{R}^{\lambda}$. To understand how and why this is done, let $Y^{=}$denote the set $f(Z)=\{f(z) \mid z \in Z\}$, and let

$$
Y^{s}=\left\{y \in \mathbb{R}^{p} \mid y \leq f(z) \quad \text { for some } z \in Z\right\} .
$$

The set $Y^{\prime}$ is called the outcome set (or image) of $Z$ under $f$. The sets $Y^{=}$and $Y^{s}$ have been used to good effect by many researchers in multiple objective mathematical programming, including, for instance, Benson [10], Bitran and Magnanti [14], Dauer [16], Dauer and Liu [17], Dauer and Saleh [18] and $Y u$ [51]. Notice that in outcome space $\mathbb{R}^{p}$, if we define the efficient sets $Y_{i}^{i}$ and $Y \stackrel{\zeta}{L}$ by

$$
Y_{\bar{k}}^{\bar{i}}=\left\{\bar{y} \in Y^{=} \mid \quad \text { there is no } y \in Y^{=} \text {such that } y \geq \bar{y} \text { and } y \neq \bar{y}\right\}
$$

and

$$
Y \leq s=\left\{\bar{y} \in Y^{\leq} \mid \quad \text { there is no } y \in Y^{\leq} \text {such that } y \geq \bar{y} \text { and } y \neq \bar{y}\right\} \text {, }
$$


respectively, then $f\left(Z_{l}\right)=Y_{l}=Y_{i}$. We thus denote $Y_{i}=Y_{i}$ as simply $Y_{E}$, and we refer to $Y_{l}$ as the efficient outcome set for problem (MMP).

To effectively generate global samples of manageable sizes of points in $Z_{\boldsymbol{l}}$, the global shooting procedure finds samples of points in $Y \cong$ that belong to the efficient outcome set $Y_{l}$. The rationale for this comes from the following three observations. First, in practice, the number of objective functions $p$ in problem (MMP) is generally much smaller than the number of decision variables $k$. As a result, the size and structure of $Y^{=}$is invariably much smaller and simpler than $Z[17,18,42]$. Second, frequently many points in $Z_{F}$ are mapped by $f$ onto a single outcome in $Y_{l}$ [3,10,16-18]. Generating samples of points directly from $Y_{l}$ thus avoids risking redundancies of calculations that could occur if points from $Z_{l}$ : were generated instead. Finally, it has been shown that, in practice, decision makers base their decisions on outcome space considerations rather than on decision space considerations [17, 18,42].

Another important characteristic of the global shooting procedure is its generality. Stated another way, the prerequisites for using the procedure are relatively minimal. Essentially, the only requirement is that $Y_{R}$ be contained in some compact subset of the set $Y^{<}$. As a result. the global shooting procedure can be used on a wide variety of problems (MMP). including, for instance, multiple objective linear, quadratic, convex, and even nonconvex programming problems.

There are a number of sets of assumptions under which $Y_{t}$ is guaranteed to be contained in a compact subset of $Y^{*}$. For instance, this will hold if the outcome set $Y=f(Z)$ is compact. More generally, however, we may state weaker sufficient conditions for using the global shooting procedure.

In particular, we will assume in the sequel that the following two relatively mild requirements are fulfilled. First, the set $Y^{\leq}$must be $\mathbb{R}_{+}^{\prime \prime}$-compact; that is, for each $y \in Y^{*}, Y^{*}$ $\cap\left(\{y\}+\mathbb{R}_{+}^{\prime \prime}\right)$ must be a compact set, where $\mathbb{R}_{+}^{\prime \prime}$ denotes $\left\{\lambda \in \mathbb{R}^{\prime \prime} \mid \lambda \geq 0\right\}$ (cf. [5I]). Second, there must exist a point $\hat{y} \in \mathbb{R}^{\prime \prime}$ such that $y \geq \hat{y}$ for all $y \in Y_{l}$. Taken together, these two requirements imply that $Y_{l} \neq \phi[51]$, and that $Y_{l}$ is a subset of the compact set $Y$ given by

$$
Y=\{y \in Y \leq \mid y \geq \hat{y}\} .
$$

The global shooting procedure generates samples of points in the compact set $Y$ that belong to $Y_{l} \subseteq Y$.

To seek a globally representative subset of $Y_{\ell:}$, the global shooting procedure begins by constructing a special simplex that contains the compact set $Y$. To accomplish this, it uses the following result ( $\mathrm{cf}$. [42. Theorem 5.2 .1 and its proof]). Let $e^{\prime} \in \mathbb{R}^{\prime \prime}$ denote the vector in which each entry is 1.0 .

THEOREM 1: Let

$$
\beta=\max \langle e, y\rangle, \quad \text { subject to } y \in Y,
$$

and, for each $i=1,2, \ldots, p$, let

$$
\beta_{i}=\min y_{i}, \quad \text { subject to } y \in Y .
$$

Define $(p+1)$ points $v^{\prime} \in \mathbb{R}^{\prime \prime}, j=0,1, \ldots, p$, according to the formulas 


$$
v_{i}^{0}=\beta_{i}, \quad i=1,2, \ldots, p
$$

and, for each $j=1,2, \ldots, p$,

$$
v_{i}^{j}= \begin{cases}v_{i}^{0}, & \text { if } i \neq j, \\ \beta+\beta_{j}-\left\langle e, v^{0}\right\rangle, & \text { if } i=j .\end{cases}
$$

Then the convex hull $S$ of $\left\{v^{j} \mid j=0,1, \ldots, p\right\}$ is either a singleton or a $p$-dimensional simplex, and $S$ contains $Y$.

If the set $S$ in Theorem 1 is a singleton, then $S=\left\{v^{0}\right\}$. In this case, because $S \supseteq Y \supseteq Y_{E}$, it follows that $Y_{E}=\left\{v^{0}\right\}$, so that the goal of representing $Y_{E}$ is complete. We shall therefore assume in the following that $S$ is not a singleton.

The importance of the simplex $S$ in the global shooting procedure is shown in the next result. To present this result, let $S^{0}$ denote the subsimplex of $S$ given by the convex hull of $\left\{v^{j} \mid j=1,2, \ldots, p\right\}$. Also, for each $d \in S^{0}$, define the mathematical programming problem $\left(P_{d}\right)$ by

$$
\begin{gathered}
\left(P_{d}\right) \max \left\langle e, v^{0}+\alpha\left(d-v^{0}\right)\right\rangle, \\
\text { subject to } v^{0}+\alpha\left(d-v^{0}\right) \in Y, \quad \alpha \geq 0,
\end{gathered}
$$

where $\alpha \in \mathbb{R}$ is the only variable. Then we may state the result as follows.

THEOREM 2: A vector $y^{*}$ belongs to $Y_{t:}$ only if for some $d \in S^{0}, y^{*}=v^{0}+\alpha^{*}(d-$ $\left.v^{0}\right)$, where $\alpha^{*}$ is an optimal solution for problem $\left(P_{d}\right)$.

For a proof of Theorem 2, see [42, Theorem 5.2.2]. Theorem 2 guarantees that if problem $\left(P_{d}\right)$ were solved for each $d \in S^{0}$, then all of $Y_{l}$ would be obtained. However, problem $\left(P_{d}\right)$ cannot generally be solved for every $d \in S^{0}$. The global shooting procedure will instead seek global representations of $Y_{t}$ by solving problem $\left(P_{t}\right)$ for each $d$ chosen from various samples of points in $S^{0}$. For each $d \in S^{0}$ chosen, solving $\left(P_{d}\right)$ amounts to shooting from the vertex $v^{0}$ of $S$ in the direction $\left(d-v^{0}\right)$ toward the subsimplex $S^{0}$ of $S$ as far as possible while remaining in $Y$. Because $S^{0}$ is a $(p-1)$-dimensional simplex with known vertices $v^{j}, j=1,2, \ldots, p$, many possible types of samples of points from $S^{0}$ can be constructed [42].

In practice, solving problem $\left(P_{d}\right)$ for a given $d \in S^{0}$ is hampered by the presence of $Y$ in the formulation. It is easy to show, however, that in general, as a more practical alternative, for any given $d \in S^{0}$, one may instead solve the equivalent problem $\left(\dot{P}_{d}\right)$ given by

$$
\begin{gathered}
\left(\hat{P}_{d}\right) \max \alpha, \\
\text { subject to } f(z)-\left(d-v^{0}\right) \alpha \geq v^{0}, \quad z \in Z, \quad \alpha \geq 0
\end{gathered}
$$

(cf. [42, Theorem 5.2.3]).

For a given $y^{*} \in Y_{t}$, Theorem 2 states that there must exist a point $d$ in the simplex $S^{0}$ that generates $y^{*}$ in the sense that when problem $\left(P_{d}\right)$ is solved for an optimal solution $\alpha^{*}$, $y^{*}$ can be found via the equation 


$$
y^{*}=v^{0}+\alpha^{*}\left(d-v^{0}\right)
$$

In fact, from the proofs of Theorems 1 and 2, it can be shown that for each $y^{*} \in Y_{l}$, the vector $d$ that yields $y^{*}$ via (2) in this way is unique in $S^{(0)}$ ( see [42. Theorem 5.2.4]).

Unfortunately, the condition in Theorem 2 for $y^{*} \in Y_{\ell}$ : to hold is a necessary, but not sufficient, one. In other words, if $d \in S^{0}$ is arbitrarily chosen, then attempting to find an optimal solution $\alpha^{*}$ to problem $\left(P_{d}\right)$ and using (2) may fail to produce a point $y^{*} \in Y_{t}$.

In particular, for each $d \in S^{0}$, there are three possibilities for problem $\left(P_{d}\right)$. First, problem $\left(P_{d}\right)$ may have an optimal solution $\alpha^{*}$ that yields a point $l^{*} \in Y_{l}$ via (2). Second, an optimal solution $\alpha^{*}$ may exist, but the point $y^{*}$ that it yields via (2) may be a boundary point of $Y$ that does not belong to $Y_{l}$. Third, problem $\left(P_{d}\right)$ may be infeasible. Ideally, we would like to avoid generating points $d \in S^{0}$ for which the second and third possibilities occur. The global shooting procedure cannot always avoid these possibilities. However, it often handles them in a reasonably efficient way. The next two theorems will help explain when these possibilities can occur and how the procedure handles them.

Let $D=\left\{d \in S^{0} \mid\right.$ Problem $\left(P_{d}\right)$ is feasible $\}$. By the following result, in certain situations, for a given $d \in S^{0}$, the possibility that problem $\left(P_{d}\right)$ is infeasible does not exist.

\section{THEOREM 3:}

(i) If $Y$ is a convex set, then $D$ is also a convex set.

(ii) If $v^{\prime \prime} \in Y^{*}$, then $D=S^{\prime \prime}$.

Theorem 3 follows from [42, Remark 5.2.4 and Theorem 5.2.5]. From [51], it is easy to see that the most common case of problem (MMP) in which $Y$ is guaranteed to be a convex set is when $Z$ is convex and, for each $j=1,2, \ldots, p, f$ is a concave function on $Z$ [in this case, problem (MMP) is a multiple objective convex programming problem]. Thus, from part (i) of Theorem 3, for any multiple objective convex programming problem. if problem $\left(P_{d}\right)$ is feasible for $d=d^{1} \in S^{0}$ and for $d=d^{2} \in S^{0}$, then problem $\left(P_{d}\right)$ must also be feasible for any vector $d$ that is a convex combination of $d^{1}$ and $d^{2}$.

The condition in part (ii) of Theorem 3 that $v^{*} \in Y$ is sufficient to guarantee that $D=$ $S^{\prime \prime}$, even if $Y$ is not a convex set. Although we know of no general classes of problem (MMP) for which this condition is guaranteed to hold, part (ii) of the theorem can be used in many individual cases to help choose appropriate algorithms to solve problem $\left(P_{d}\right)$.

Whenever a vector $d \in S^{0}$ chosen in the global shooting procedure yields a feasible problem $\left(P_{d}\right)$. the procedure finds an optimal solution $\alpha^{*}$ to the problem. From $\alpha^{*}$ and (2), it then generates a point $y^{*}$ on the boundary of $Y$. Because $y^{*} \notin Y_{V}$ is possible, the point $y^{*}$ must be tested for efficiency. The next result provides the basis for this test. Fortunately, when $y^{*} \notin Y_{l}$, the test generates a point in $Y_{E}$ that dominates $y^{*}$. The proof of this result follows easily from Benson [8]. Let int $\mathbb{R}_{+}^{\prime \prime}=\left\{\lambda \in \mathbb{R}^{p} \mid \lambda>0\right\}$.

THEOREM 4: Assume that $\lambda \in \operatorname{int} \mathbb{R}_{+}^{\prime \prime}$ and $\bar{y} \in Y$. Then $\bar{y}$ belongs to $Y_{l}$ if and only if $\bar{y}$ is an optimal solution to the problem $\left(P_{\bar{y}, \lambda}\right)$ given by

$$
\begin{gathered}
\left(P_{i, \lambda}\right) \max \langle\lambda, y\rangle \\
\text { subject to } y \geq \bar{y}, \quad y \in Y .
\end{gathered}
$$


Furthermore, problem $\left(P_{\bar{j}, \lambda}\right)$ always has at least one optimal solution, and any optimal solution $\hat{y}$ for problem $\left(P_{\bar{j}, \lambda}\right)$ satisfies $\hat{y} \in Y_{E}$.

We may now state the global shooting procedure for problem (MMP) as follows.

STEP 1. 1.1. Construct the $p$-dimensional simplex $S$ containing $Y$ as described in Theorem 1.

1.2. Generate a discrete sample $d^{i}, i=1,2, \ldots, q$, of points from the subsimplex $S^{0}$ of $S$.

STEP 2. 2.1. Set $i=1$ and $R Y_{t:}=\varnothing$.

2.2. Let $d=d^{i}$. If problem ( $\left.P_{d}\right)$ is infeasible, go to Step 2.5. Otherwise, find the optimal value $\alpha^{*}$ of problem $\left(P_{d}\right)$ and continue.

2.3. Let $y^{i}=v^{0}+\alpha^{*}\left(d^{i}-v^{0}\right)$.

2.4. Let $\bar{y}=y^{i}$ and $\lambda=e$. Find any optimal solution $\hat{y}$ to the problem $\left(P_{\bar{y}, \lambda}\right)$. Set $R Y_{t:}=R Y_{\ell:} \cup\{\hat{y}\}$.

2.5. Set $i=i+1$. If $i \leq q$, go to Step 2.2. Otherwise, STOP: $R Y_{t:}$ is the discrete representation of $Y_{t}$ returned by the procedure.

Let $S S^{0}$ denote the sample of points generated from the subsimplex $S^{0}$ of $S$ in Step 1.2 of the procedure. For each point $d \in S S^{0}$, Step 2.2 of the procedure examines whether or not problem $\left(P_{d}\right)$ is feasible. If it is infeasible, then the procedure rejects the point $d$ and draws another point from $S S^{\circ}$. But if problem $\left(P_{d}\right)$ is feasible, then it is solved, and its optimal value $\alpha^{*}$ is used in Step 2.3 to find the point $y^{i}$ on the boundary of $Y$. Intuitively, the process of obtaining $y^{i}$ in this way corresponds to shooting from $v^{0}$ in the direction ( $d$ $-v^{0}$ ) until the boundary of $Y$ is hit at a point $y^{i}$. The goal of this shooting process is to obtain a point $y^{i}$ that satisfies $y^{i} \in Y_{\ell}$. The test of whether or not this condition is satisfied occurs in Step 2.4. In particular, from Theorem 4, $y^{\prime} \in Y_{\ell}$ if and only if the point $\hat{y}$ found in Step 2.4 equals $y^{i}$. In this case, $y^{i}$ is added to the discrete sample $R Y_{l}$ of $Y_{l}$. If $\hat{y} \neq y^{i}$, then $\hat{y}$ is added to $R Y_{l}$ because, in this case, by Theorem 4, $y^{i} \notin Y_{l}$ and $\hat{y} \in Y_{l}$.

The global shooting procedure thus reduces the issue of generating a sample of points from $Y_{i}$ to the simpler issue of generating a sample of points $S S^{0}$ from the simplex $S^{0}$ opposite to $v^{0}$ in $S$. By Theorem 2, in this way the potential exists to globally represent $Y_{L}$, no matter how vast or complicated the set $Y_{I}$ is.

ILLUSTRATION: To illustrate the mechanics of the global shooting procedure, consider the multiple objective mathematical program (MMP) where $Z$ is the unit hypercube in $\mathbb{R}^{10}$; that is,

$$
Z=\left\{z \in \mathbb{R}^{10} \mid 0 \leq z_{i} \leq 1, i=1,2, \ldots, 10\right\}
$$

$p=2$, and $f_{1}(z)$ and $f_{2}(z)$ are the linear functions given by

$$
f_{1}(z)=-\sum_{i=1}^{4} z_{i}+\sum_{i=5}^{8}(0.667) z_{i}-\sum_{i=9}^{10}(0.750) z_{i},
$$

and 


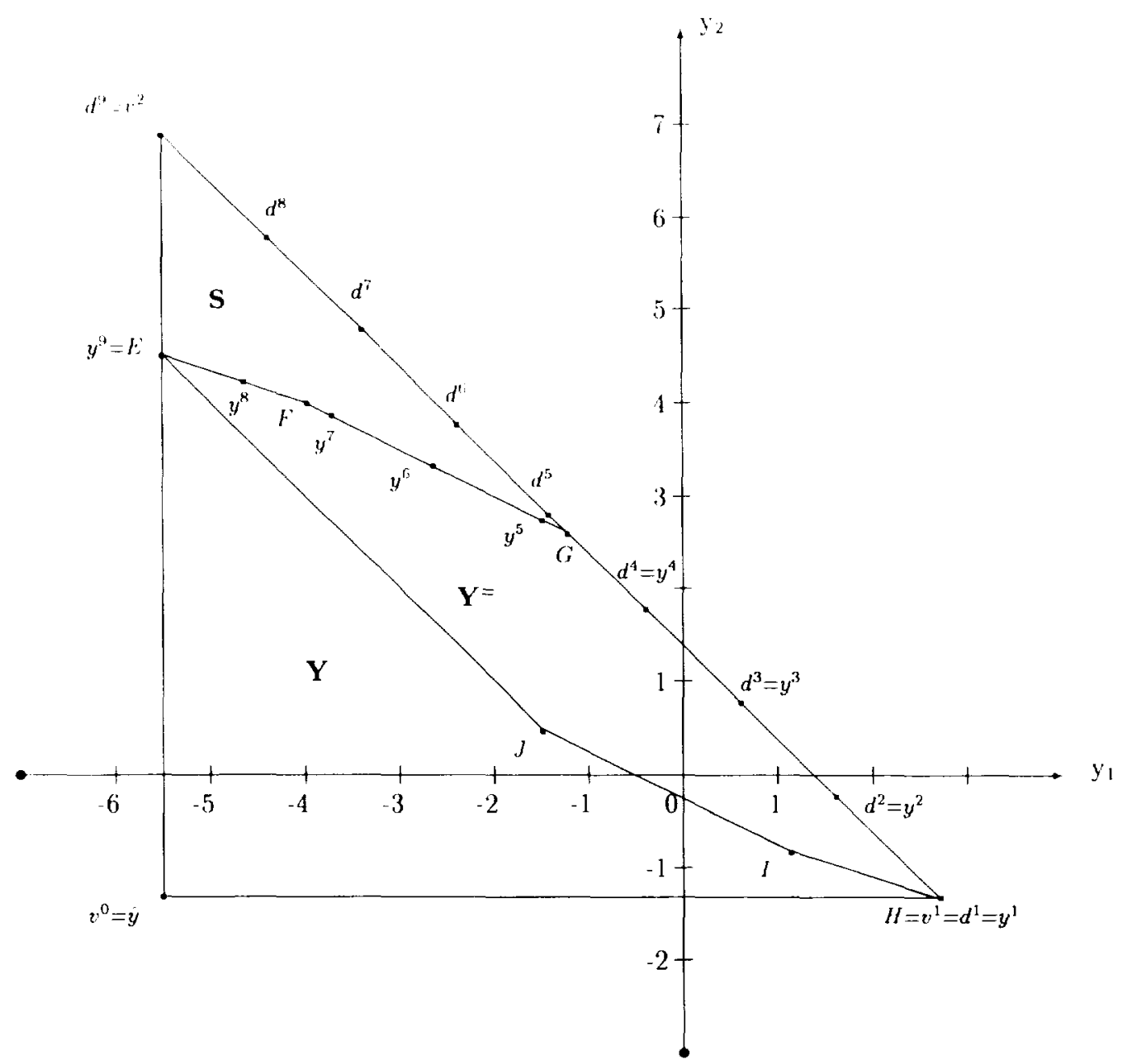

Figure 1. Graph for illustration.

$$
f_{2}(z)=\sum_{i=1}^{4} z_{i}-\sum_{i=5}^{8}(0.333) z_{i}+\sum_{i=9}^{10}(0.250) z_{i},
$$

respectively, for each $z \in Z$. Then problem (MMP) is a multiple objective linear programming problem. By examining the extreme points and faces of $Z$, it is not difficult to show that $Z$ has 34 efficient extreme points, 68 efficient edges, one two-dimensional efficient face, and two four-dimensional efficient faces. In contrast, the outcome set $Y^{=}=$ $f(Z)$ is a simple two-dimensional, compact polyhedron in $\mathbb{R}^{2}$ with only four efficient extreme points and three efficient edges.

In general, of course, it is impossible or, at best, quite burdensome computationally to obtain $Y^{-}$explicitly, even in the case of multiple objective linear programming [18]. However, we have chosen a special illustrative example wherein $\gamma$ can be obtained explicitly to enable us to demonstrate the steps of the global shooting procedure as graphically as possible.

In particular, the outcome set $Y^{=}$, shown in Figure 1, is the six-sided, two-dimensional 
Table 1. Key to coordinates.

\begin{tabular}{lc}
\hline Point & $\begin{array}{c}\text { Coordinates } \\
\left(y_{1}, y_{2}\right) \text { in } Y^{-}\end{array}$ \\
\hline$E$ & $(-5.500,4.500)$ \\
$F$ & $(-4.000,4.000)$ \\
$G$ & $(-1.333,2.667)$ \\
$H$ & $(2.667,-1.333)$ \\
$I$ & $(1.167,-0.833)$ \\
$J$ & $(-1.500,0.500)$ \\
\hline
\end{tabular}

polyhedron with extreme points $E, F, G, H, I$, and $J$. The coordinates of these extreme points are given in Table 1 . The efficient outcome set $Y_{l}$ consists of the union of the three line segments $\overline{E F}, \overline{F G}$, and $\overline{G H}$.

Because $Y^{=}$is compact, it follows immediately that $Y^{\leq}$is $\mathbb{R}_{+}^{2}$-compact. Also, because $y$ $\geq \hat{y}$ for all $y \in Y^{*}$, where

$$
\hat{y}_{1}=-5.500=\min _{z \in \%} f_{1}(z)
$$

and

$$
\hat{y}_{2}=-1.333=\min _{z \in Z} f_{2}(z)
$$

we know that $y \geq \hat{y}$ for all $y \in Y_{E}$. Thus, with this choice of $\hat{y}$, the two prerequisites for using the global shooting procedure on this illustrative problem are fulfilled. Notice from (1) that with this choice of $\hat{y}$, the compact set $Y$ containing $Y_{E}$ in this problem is the compact polytope given by the convex hull of the five points $E, F, G, H$, and $\hat{y}$ (cf. Figure 1).

The global shooting procedure will generate a discrete sample of points in $Y$ that belongs to $Y_{E}$. The particular sample obtained depends upon various factors, including, in particular, the choice made in Step 1.2 for the discrete sample of points $S S^{0}$ in the subsimplex $S^{0}$ of $S$ (cf. Section 4 below). What follows is a step-by-step summary of the calculations and outputs that the global shooting procedure gives for this illustrative problem under one choice for $S S^{0}$.

In Step 1 of the global shooting procedure, a two-dimensional simplex $S$ containing $Y$ must first be constructed via Theorem 1 . To accomplish this, the values of $\beta, \beta_{1}$, and $\beta_{2}$ defined in Theorem 1 must be found. From the definitions of $Y$ and of $\beta, \beta_{1}$, and $\beta_{2}$, these three values can be found by maximizing $\left(y_{1}+y_{2}\right)$, minimizing $y_{1}$, and minimizing $y_{2}$, respectively, each subject to the linear constraints

$$
f(z) \geq y \geq \hat{y}, \quad z \in Z .
$$

Solving the resulting three linear programming problems yields the values $\beta=1.333, \beta_{1}=$ -5.500 , and $\beta_{2}=-1.333$. From Theorem 1 , the simplex $S$ is then given by the convex hull of $v^{j}, j=0,1,2$, where 
Table 2. Step 2 results for illustration.

\begin{tabular}{ccccc}
\hline Execution no. $i$ & Sample point $d^{i}$ & Value of $\alpha^{*}$ & Coordinates of $y^{\prime}$ & $\begin{array}{c}\text { Point } \hat{y} \text { added } \\
\text { to } R Y_{L}\end{array}$ \\
\hline 1 & $(2.667,-1.333)$ & 1.000 & $(2.667,-1.333)$ & $(2.667,-1.333)$ \\
2 & $(1.646,-0.313)$ & 1.000 & $(1.646,-0.313)$ & $(1.646,-0.313)$ \\
3 & $(0.625,0.708)$ & 1.000 & $(0.625,0.708)$ & $(0.625,0.708)$ \\
4 & $(-0.396,1.729)$ & 1.000 & $(-0.396,1.729)$ & $(-0.396,1.729)$ \\
5 & $(-1.417,2.750)$ & 0.993 & $(-1.444,2.722)$ & $(-1.444,2.722)$ \\
6 & $(-2.438,3.771)$ & 0.917 & $(-2.692,3.346)$ & $(-2.692,3.346)$ \\
7 & $(-3.458,4.792)$ & 0.851 & $(-3.762,3.881)$ & $(-3.762,3.881)$ \\
8 & $(-4.479,5.813)$ & 0.779 & $(-4.705,4.235)$ & $(-4.705,4.235)$ \\
9 & $(-5.500,6.833)$ & 0.686 & $(-5.500,4.500)$ & $(-5.500,4.500)$ \\
\hline
\end{tabular}

$$
v^{0}=\left[\begin{array}{l}
-5.500 \\
-1.333
\end{array}\right], \quad v^{1}=\left[\begin{array}{c}
2.667 \\
-1.333
\end{array}\right], \quad v^{2}=\left[\begin{array}{c}
-5.500 \\
6.833
\end{array}\right]
$$

Simplex $S$ is also shown in Figure 1.

The second part of Step 1 calls for generating a discrete sample $d^{i}, i=1,2, \ldots, q$, of points from the subsimplex $S^{0}$ of $S$, where $S^{0}$ is given by the convex hull of $v^{1}$ and $v^{2}$. Notice in this case that $S^{0}$ is the line segment connecting $v^{1}$ and $v^{2}$. There are many possible choices for generating $S S^{0}=\left\{d^{i} \mid i=1,2, \ldots, q\right\}$. For illustrative purposes, suppose that $q=9$ and, for each $i=1,2, \ldots, 9$, let $d^{i}$ be given by the convex combination

$$
d^{i}=\left(\frac{9-i}{8}\right) v^{\prime}+\left(\frac{i-1}{8}\right) v^{2}
$$

of $v^{1}$ and $v^{2}$. Then the points $v^{1}=d^{1}, d^{2}, d^{3}, \ldots, d^{8}, d^{9}=v^{2}$ subdivide $S^{0}$ into eight line segments of equal length ( $\mathrm{cf}$. Figure 1 ).

Step 2 of the global shooting procedure is next executed $q=9$ times. In a typical execution $i \in\{1,2, \ldots 9\}$, with $d=d^{i}$, problem $\left(P_{d}\right)$ is solved. This problem, as we have seen, may be solved by solving the equivalent problem $\left(\hat{P}_{d}\right)$. In this illustration, by the definitions of $Z$ and $f$, problem $\left(P_{d}\right)$ is a linear program in the nonnegative variables $\left(\alpha, z^{T}\right) \in \mathbb{R}^{1 !}$ with two constraints and simple upper bounds on $z$.

Recall that for each $d=d^{\prime}$, the idea in solving problem $\left(P_{d}\right)$ is, starting at $v^{0}$, to shoot in the direction ( $d-v^{0}$ ) toward the subsimplex $S^{0}$. If $Y$ is never encountered in this way, problem $\left(\hat{P}_{d}\right)$ is infeasible and this execution of Step 2 is terminated. Otherwise, problem ( $\hat{P}_{d}$ ) has a finite optimal value $\alpha^{*}$, and, in Steps 2.2 and 2.3, by finding it, we shoot as far as possible from $v^{0}$ in the direction $\left(d^{i}-v^{0}\right)$ until the point $y^{i}$ on the boundary of $Y$ is found.

When problem $\left(P_{d}\right)$ successfully generates a point on the boundary of $Y$ for some $d=$ $d^{\prime}$, this point $\bar{y}$ is tested in Step 2.4 for efficiency by solving the problem $\left(P_{\bar{\zeta}, \lambda}\right)$ with $\lambda=e$. In this case, by the definition of $Y$, with $\lambda=e$, problem $\left(P_{\bar{j}, \lambda}\right)$ may be solved by maximizing $\left(y_{1}+y_{2}\right)$, subject to the linear constraints

$$
f(z) \geq y \geq \bar{y}, \quad z \in Z
$$

that is, Step 2.4 can be accomplished by solving a linear programming problem.

Table 2 summarizes the results of executing Step 2 the required nine times on this prob- 
lem. In this table, all decimals are given to three significant digits. In this case, for each $d=$ $d^{i}, i=1,2, \ldots, 9$, problem $\left(P_{d}\right)$ has an optimal solution $\alpha^{*}$ that immediately yields a point $y^{i} \in Y_{E}$. Thus, the discrete sample $R Y_{E}=\left\{y^{i} \mid i=1,2, \ldots, 9\right\}$ of points in $Y_{E}$ generated by the procedure is given by the nine distinct points in the last column of Table 2 . These points are also shown in Figure 1. Notice that because the illustrative problem is a multiple objective linear program, the optimization problems $\left(P_{d}\right)$ and $\left(P_{\bar{j}, \lambda}\right)$ with $\lambda=e$ that are solved during the global shooting procedure are all linear programming problems.

\section{SAMPLE IMPLEMENTATIONS}

The computational requirements of the global shooting procedure depend to a great extent upon the type of problem (MMP) that is being analyzed. For example, when problem (MMP) is a multiple objective convex programming problem with a compact feasible region $Z$, all of the optimization problems that must be solved to implement the procedure are either convex programming problems or concave minimizations over convex sets. Therefore, in this case standard convex programming methods $[6,35]$ can be combined with more recently developed concave minimization methods $[9,29]$ to implement the global shooting procedure. Similarly, standard methods of linear programming [5] and of integer linear programming $[38,39]$ can be used to implement the procedure when problem (MMP) is a multiple objective linear program and a multiple objective integer linear program, respectively.

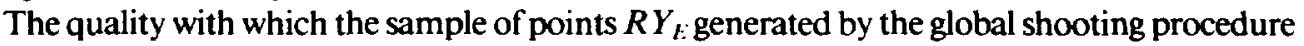
represents $Y_{t}$ seems to depend rather heavily upon the nature of the sample of points $S S^{0}$ from $S^{0}$ that it generates (cf. Step 1.2) [42]. Intuitively, it seems plausible that samples of points from $S^{0}$ that are excellent global representations of $S^{0}$ would most likely generate the best possible global representations of $Y_{t}$ attainable by the procedure, but this remains to be seen.

To illustrate some of the possible implementations of the global shooting procedure and to show how the results can vary as $S S^{0}$ varies, we will summarize the results that we obtained from four of the experimental applications that we have performed. A full report on these experimental applications can be found in [42].

Let $k=3$, and let $Z$ in problem (MMP) be the nonempty, compact polyhedron in $R^{3}$ given by

$$
Z=\left\{z \in \mathbb{R}^{3} \mid A z \leq b, z \geq 0\right\},
$$

where $A$ and $b$ are the matrix and vector defined by

$$
A=\left[\begin{array}{rrr}
6 & 15 & 10 \\
5 & 8 & 12 \\
22 & 29 & 28 \\
24 & 16 & 11 \\
1 & 0 & 4 \\
8 & 0 & 1
\end{array}\right] \quad \text { and } \quad b=\left[\begin{array}{r}
210 \\
152 \\
458 \\
312 \\
40 \\
72
\end{array}\right]
$$

respectively. In addition, let $p=3$ and, for each $j=1,2,3$, let $f$ in problem (MMP) be defined by $f_{j}(z)=z_{j}$ for each $z \in \mathbb{R}^{3}$. Then problem (MMP) is a multiple objective linear program used previously by Dessouky, Ghiassi, and Davis [19] in another context. In this example, $Y^{=}=f(Z)$ is compact, so that $Y^{s}$ is $\mathbb{R}_{+}^{3}$-compact. Because $f(z) \geq 0$ for all $z \in Z_{1 \text { : }}$ 


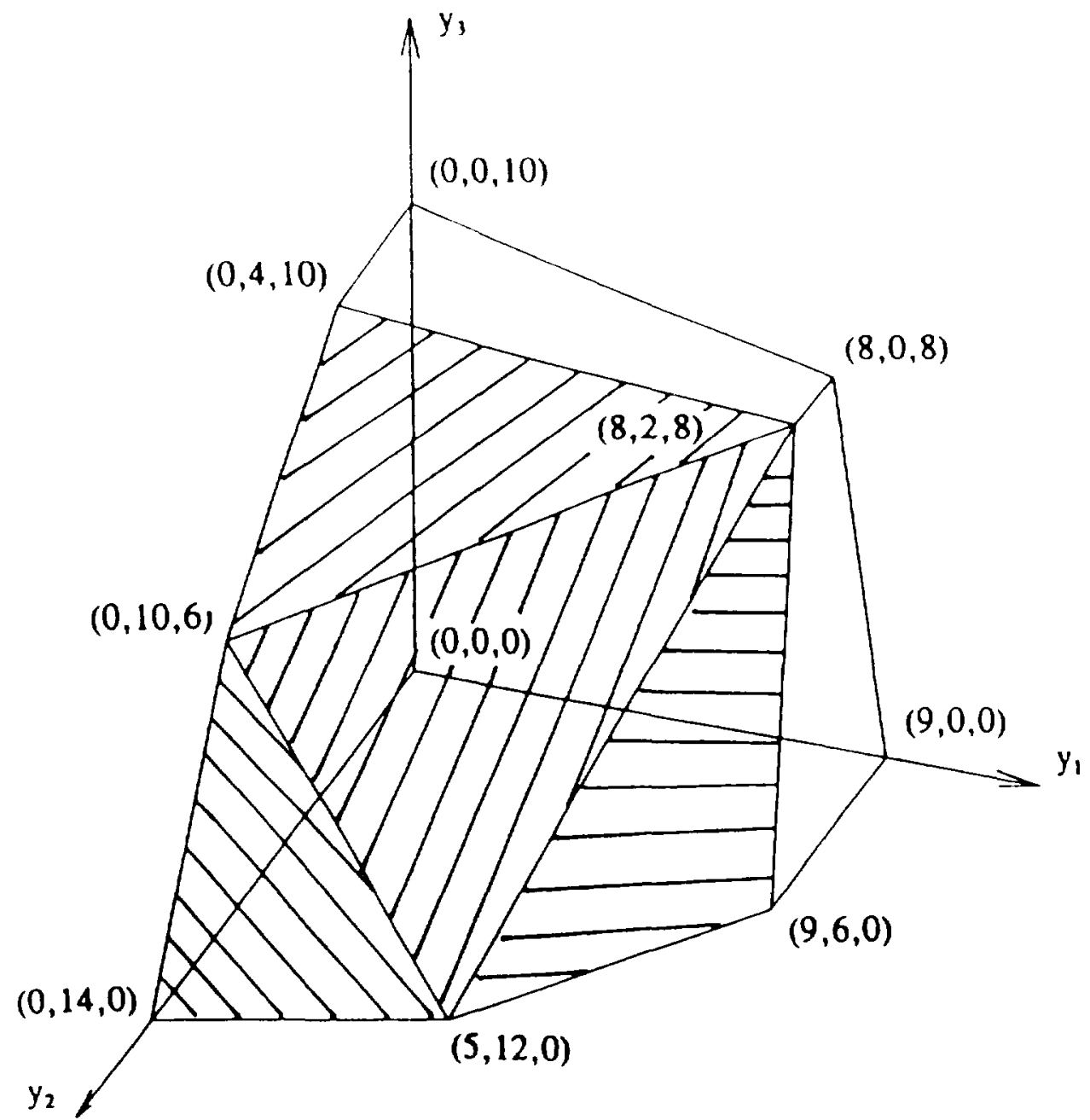

Figure 2. Graphs of $Z$ and $Y_{1}$ for example from [19].

$\subseteq Z$, we may choose $\hat{y}=0 \in \mathbb{R}^{3}$ and be guaranteed that $y \geq \hat{y}$ for all $y \in Y_{t}$. Thus, the global shooting procedure can be applied to this problem. Notice that with $\hat{y}=0$, (1) yields $Y=Z$. From [19], $Z_{I}=Y_{l}$ is known to be given by the union of the four shaded twodimensional faces of $Z$ shown in Figure 2 . Because this problem is a multiple objective linear program, we were able to use standard linear programming techniques to solve the optimizations required in the global shooting procedure.

For this example, we used the global shooting procedure four times to generate four discrete representations of $Y_{l}$. To do so, we used two different methods to generate the sample of points $S S^{\prime \prime}$ from $S^{\prime \prime}$ required by the procedure. For each method, we generated two samples, one of size 30 and one of size 50 .

The first method that we used to generate $S S^{0}$ may be called a method of random weights. This method is probabilistic in nature. We devised it by drawing upon some work of Steuer 
Table 3. Summary of experimental results.

\begin{tabular}{clcccc}
\hline $\begin{array}{c}\text { Experiment } \\
\text { no. }\end{array}$ & \multicolumn{1}{c}{$\begin{array}{c}\text { Method of } \\
\text { obtaining } S S^{\circ}\end{array}$} & $\begin{array}{c}\text { No. of distinct } \\
\text { points in } S S^{\circ}\end{array}$ & $\begin{array}{c}\text { No. of distinct } \\
\text { points in } R Y_{t}\end{array}$ & Display & Rank \\
\hline 1 & Random weights & 30 & 30 & Figure 3 & 4 \\
2 & Successive bisection & 30 & 30 & Figure 4 & 1 \\
3 & Random weights & 50 & 50 & Figure 5 & 2 \\
4 & Successive bisection & 50 & 50 & Figure 6 & 1 \\
\hline
\end{tabular}

[47]. In this method, random convex combinations of the vertices of $S^{0}$ are used to generate $S S^{0}$. In our implementation of the method, $50 \%$ of these convex combinations were drawn from a uniform distribution, and 50\% from a Weibull distribution [42].

The second method that we used to generate $S S^{0}$ may be called a method of successive ( simplicial) bisections. In global optimization, various procedures for successively partitioning a given simplex into subsimplices have been created and used to good effect [29]. We adapted one of these methods, called simplicial bisection, to the problem of generating representations $S S^{0}$ of $S^{0}$. This resulted in a deterministic method for generating such sets. In the method, $S^{0}$ is successively bisected a prechosen number of times. This results in a partition of $S^{0}$ consisting entirely of subsimplices. The set $S S^{0}$ is then generated by choosing one element from each of these subsimplices [42].

The results of these four experiments are summarized in Table 3 and Figures 3-6. In Table 3, for each representation $R Y_{t:}$ generated, rank is a number intended to help quantify the quality of the representation. It is derived subjectively and can be interpreted according to the scheme shown in Table 4. Notice that it is desirable for a representation of $Y_{E}$ to provide a good coverage of $Y_{L}$ without significant clustering. Therefore, in this ranking scheme, lower-numbered ranks are superior to higher-numbered ones. Although this ranking procedure is not precise, it may aid in summarizing at least the relative quality of alternate representations. Other possibilities exist for measuring the quality of representations of $Y_{E}$, but we will not stop to examine this issue here $[2,42]$.

Notice from Table 3 that in each of the four experiments, for each sample point $d$ in $S S^{0}$, the global shooting procedure generated a distinct point in $Y_{L}$. In this problem, $Y$ is a compact, convex set, and $\left(v^{0}\right)^{r}=(0,0,0) \in Y^{*}$. Therefore, by Theorems 3 and 4 and Steps 2.2-2.5 of the procedure, for each $d \in S S^{0}$, the global shooting procedure is guaranteed to generate a point in $Y_{\ell}$. However, the fact that in each of the four runs, each point in $S S^{0}$ generated a distinct point in $Y_{E}$ is a fortuitous result that could not have been predicted. Based on sheer numbers of points in $R Y_{E}$, the four runs are indistinguishable.

Also notice from Figures 3-6 that in each run, the representation $R Y_{t}$ of $Y_{t:}$ generated is not limited to certain types of locations in $Y$. For instance, unlike in many existing vector maximization methods for multiple objective linear programming, the points in $R Y_{\ell}$ are not arbitrarily confined to extreme points or edges of $Y_{l}$.

Table 4. Key to ranks.

\begin{tabular}{ccl}
\hline Rank & Coverage & Clustering \\
\hline 1 & Good & Insignificant \\
2 & Good & Significant \\
3 & Poor & Insignificant \\
4 & Poor & Significant \\
\hline
\end{tabular}




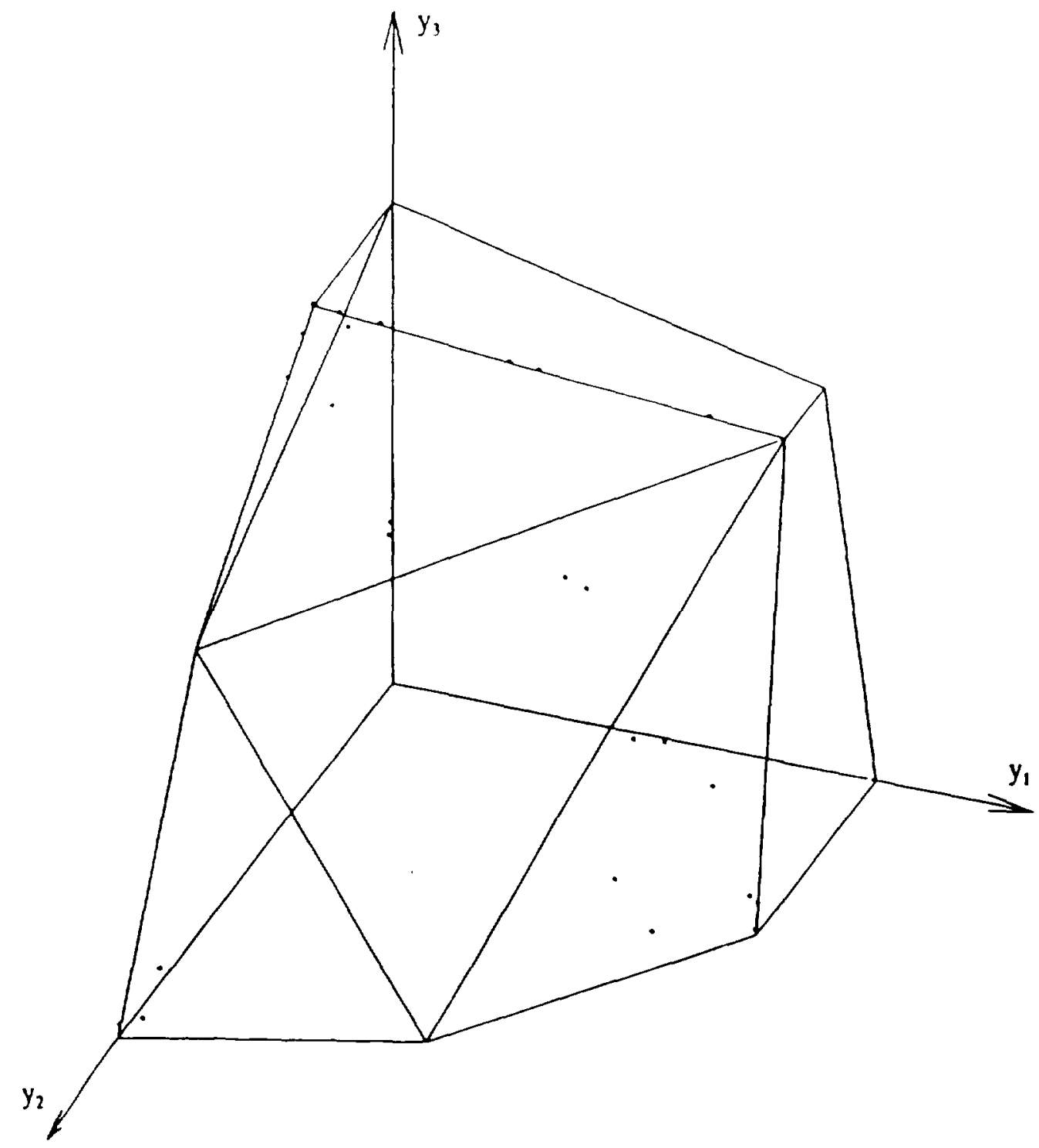

Figure 3. Graph of $R Y_{l}$ for Experiment No. 1.

However, according to the rank column of Table 3, and from Figures 3-6, the quality of the four experimental representations of $Y_{l}$ varies significantly. In particular, for this problem. the runs in which $S S^{\prime \prime}$ were generated by the method of successive bisections seemed subjectively to generate global representations of $Y_{I}$ superior to those generated by the method of random weights. This superiority appears to have been achieved both in the coverage of $Y_{t}$ and in the amount of clustering.

We caution that no precise conclusions regarding the computational abilities of the global shooting procedure should be drawn from these four applications. There are a number of reasons for this. not the least of which concerns the issue of evaluation. In particular, 


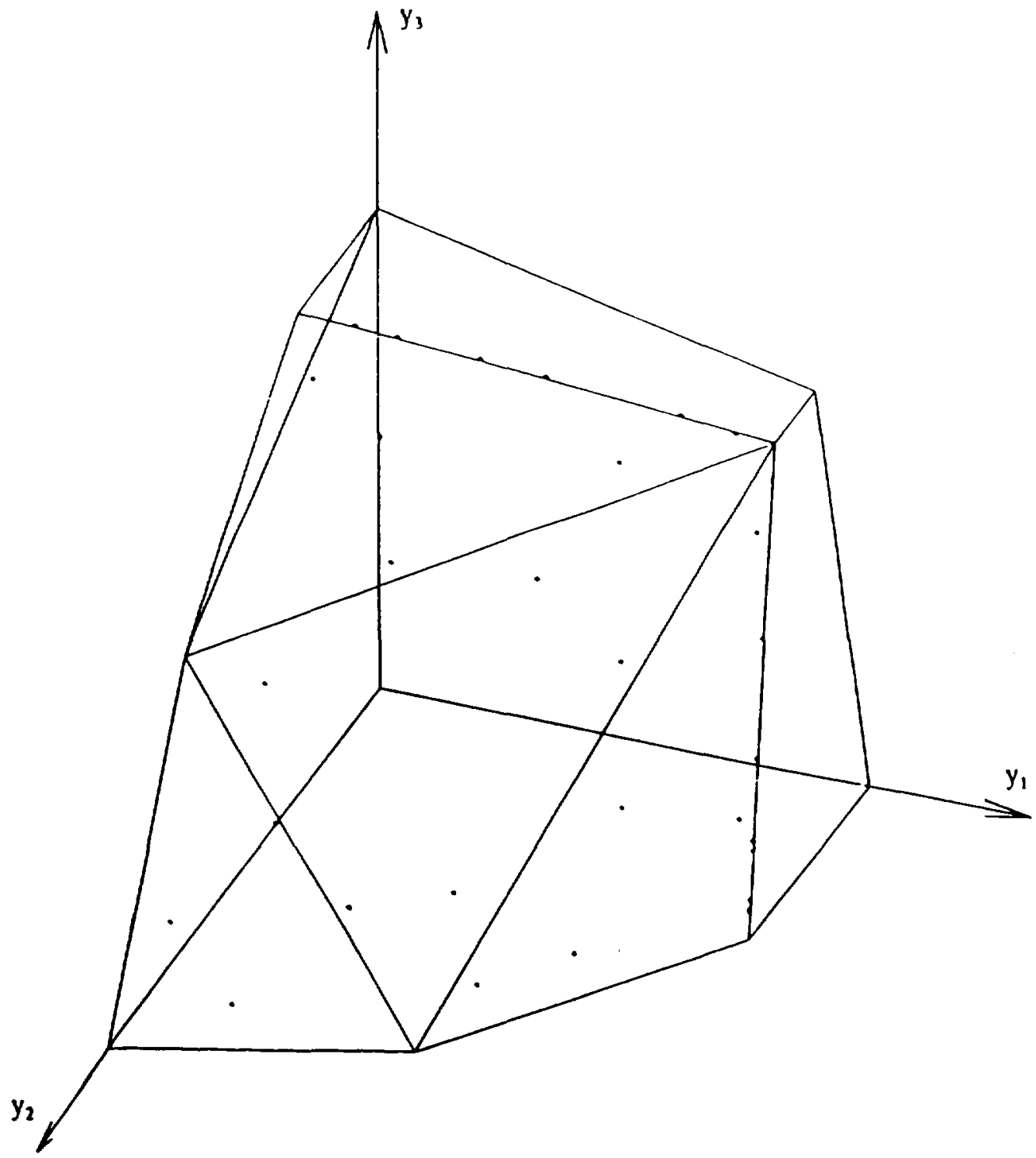

Figure 4. Graph of $R Y_{t}$ for Experiment No. 2.

before any method such as the global shooting procedure can be evaluated, it is essential that means be developed for assessing the quality of the representations of the efficient sets that are obtained for various problems, including for problems whose outcome spaces are of dimensions larger than three [42].

\section{CONCLUSIONS AND DIRECTIONS FOR FURTHER RESEARCH}

Current procedures for implementing the three major approaches for solving problem (MMP) are not wholly adequate. This is because these procedures either make overly re- 


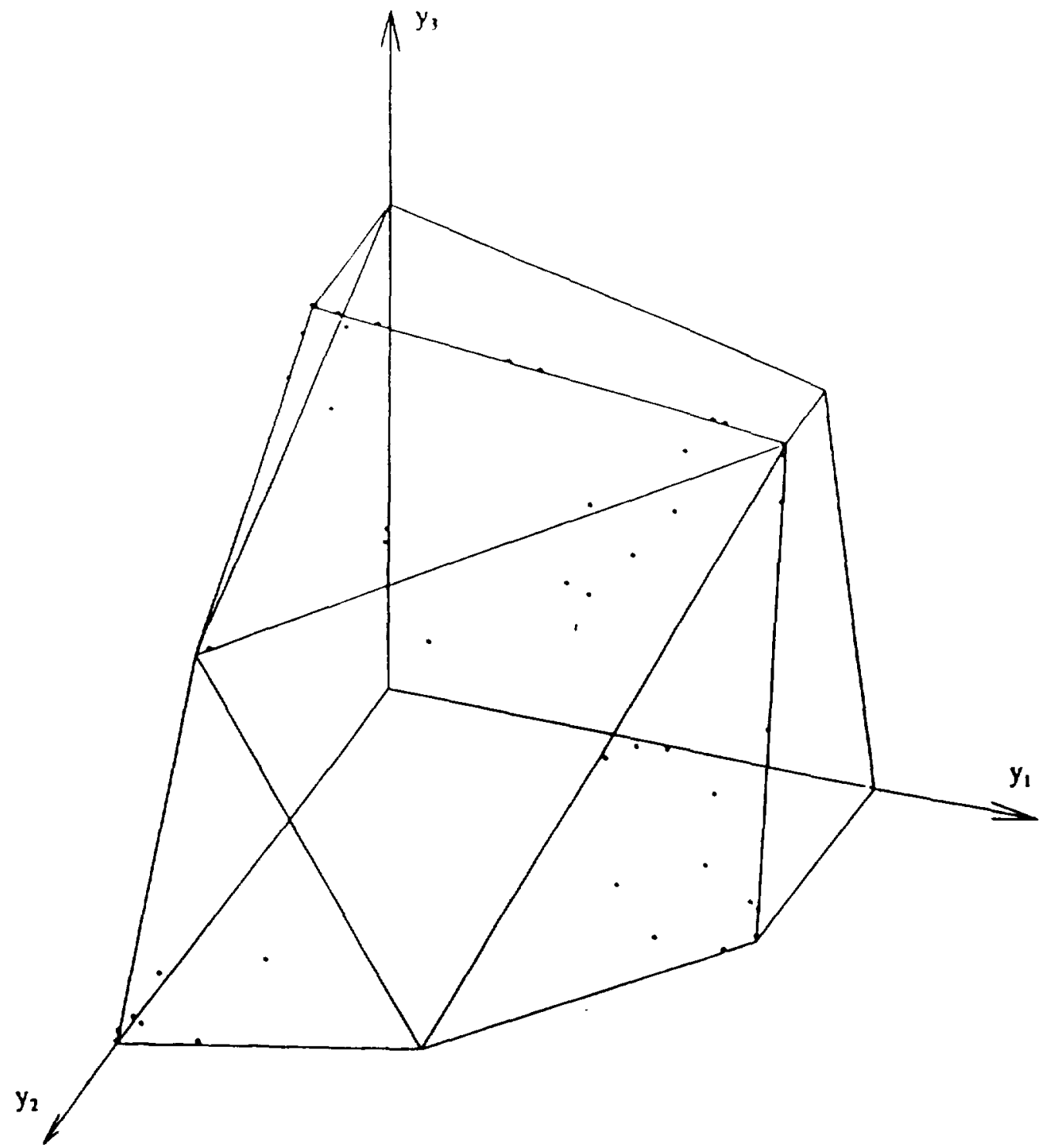

Figure 5. Graph of $R Y_{1}$ for Experiment No. 3

strictive assumptions, demand more preference information than the DM can provide, call for excessive computation. or fail to adequately globally represent the efficient set of the problem. A potential means of alleviating many of these weaknesses is to focus on a goal of generating truly global representations of the efficient set $Z_{1}$ of problem (MMP).

The global shooting procedure that we have recently formulated illustrates the potential benefits of seeking global representations of $Z_{l}$. It takes a global. rather than a local, approach, so that it has the potential to provide the DM a means of truly learning about the entire efficient set without the complications of bookkeeping, degeneracy, and exponential 


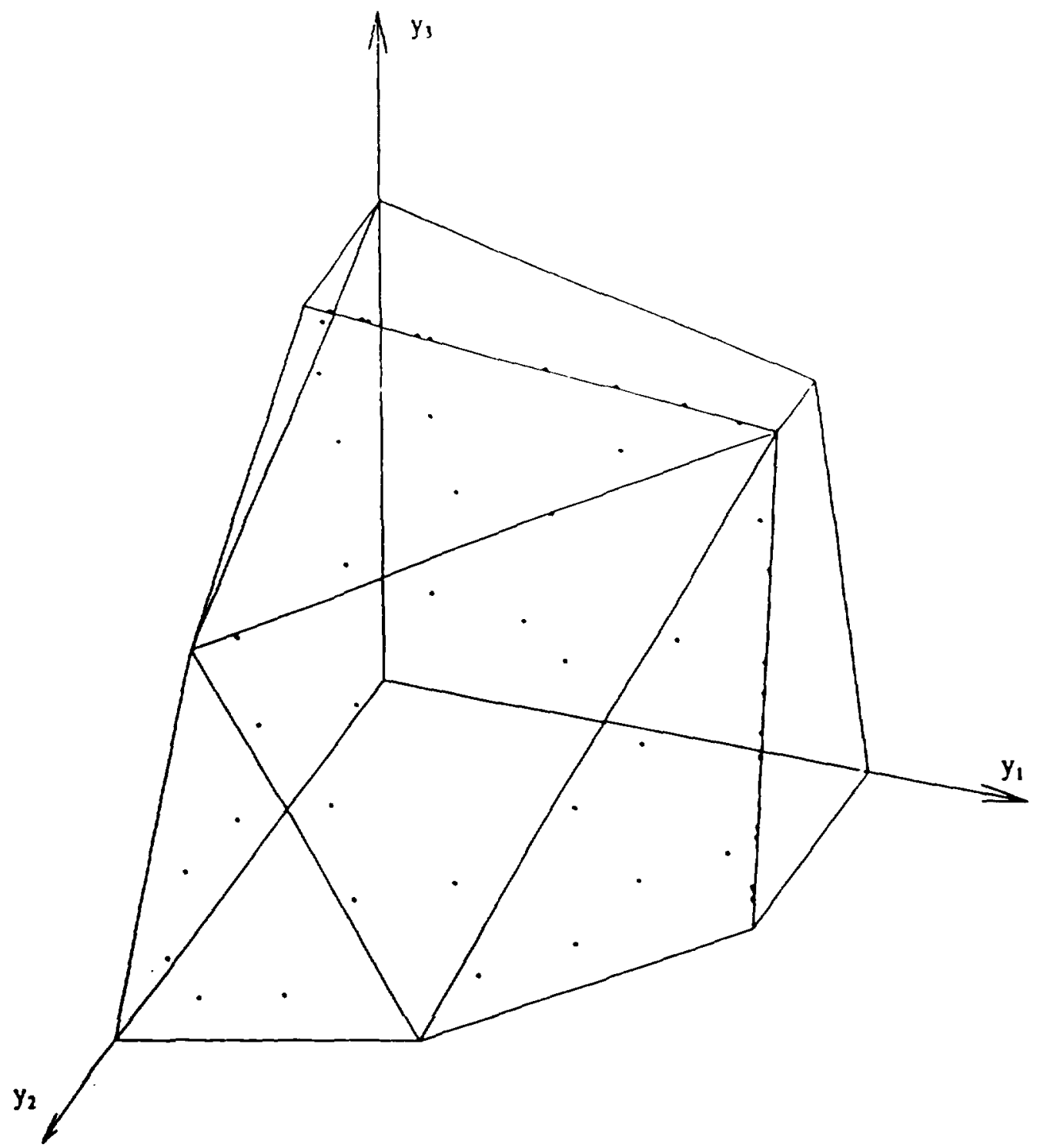

Figure 6. Graph of $R Y_{1}$ for Experiment No. 4.

growth in computational effort that have been observed with local approaches. Furthermore, approaches such as the global shooting procedure could be used without requiring the DM to provide complicated preference information or to endure tiresome iterations with the computer. Instead, the DM must agree only that his or her preferences dictate that more is better. In addition, by working in the outcome space rather than in the decision space, approaches such as the global shooting procedure could reduce the computational effort required to learn about $Z_{t}$, decrease the likelihood of overwhelming the $\mathrm{DM}$, and decrease the likelihood of generating clusters of similar points in the representation of $Z_{L}$ : 
The global shooting procedure also illustrates that the potential exists to construct global representations of $Z_{1}$ for a variety of cases of problem (MMP). This is in part because the approach of the procedure is applicable, for instance, to any problem (MMP) with a compact outcome set or, more generally, to problems (MMP) where the efficient outcome set $Y_{I}$ is compact. Furthermore, recent advances in global optimization have created a wide variety of new techniques for solving nonconvex problems [29]. It therefore seems that many other approaches based on global optimization ideas besides the global shooting procedure approach can potentially be formulated for generating global representations of $Z_{l}$ for the various cases of problem (MMP).

The set $Z_{I}$ is generally a complex, nonconvex set. Therefore, to find practical methods of generating global representations of $Z_{l}$, much work will have to be done. Perhaps the most important tasks to be accomplished are as follows.

1. Rigorous definitions of truly global representations of the efficient set of problem (MMP) must be found.

2. Methods of quantifying and comparing the quality of alternate global representations of $Z_{t}$ : must be formulated.

3. Practical means of generating adequate global representations of $Z_{1}$ for realworld problems must be found. Ideally, these representations should avoid redundancies, represent the entire set $Z_{k}$, and not overwhelm the DM.

It is hoped that the observations and results that we have provided will serve as a stimulant toward the achievement of these goals.

\section{REFERENCES}

[1] Aksoy, Y., "Interactive Multiple Objective Decision Making: A Bibliography (1965-1988)," Management Research News, 13, 1-8 (1990).

[2] Aldenderfer, M.S., and Blashfield, R.K., "Cluster Analysis," Sage University Paper Series on Quantitative Applications in the Social Sciences, Series No. 07-044, Beverly Hills, CA, 1986.

[3] Armann, R., "Solving Multiobjective Programming Problems by Discrete Representation." Optimization, 20, 483-492 (1989).

[4] Bagchi, U., "Simultaneous Minimization of Mean and Variation of Flow Time and Waiting Time in Single Machine Systems," Operations Research, 37, 118-125 (1989).

[5] Bazaraa. M.S., Jarvis. J.J.. and Sherali, H.D., Linear Programming and Network Flows, Wiley. New York, 1990.

[6] Bazaraa, M.S., Sherali, H.D., and Shetty, C.M., Nonlinear Programming: Theory and Algorithms (2nd ed.), Wiley, New York, 1993.

[7] Benson, H.P.. "Vector Maximization with Two Objective Functions," Journal of Optimization Theory and Applications, 28, 253-257 (1979).

[8] Benson, H.P., "Efficiency and Proper Efficiency in Vector Maximization with Respect to Cones," Journal of Mathematical Analysis and Applications. 93, 273-289 (1983).

[9] Benson, H.P.. "Concave Minimization: Theory, Applications and Algorithms," in R. Horst and P. Pardalos (Eds.), Handbook of Glohal Optimization. Kluwer Academic Publishers, Dordrecht, 1995, pp. 43-148.

[10] Benson, H.P., "A Geometrical Analysis of the Efficient Outcome Set in Multiple Objective Convex Programs with Linear Criterion Functions," Journal of Glohal Optimization, 6, 231251 (1995).

[11] Benson, H.P., Lee, D., and McClure, J.P., "Applying Multiple Criteria Decision Making in Practice: The Citrus Rootstock Selection Problem in Florida," Discussion Paper, Department of Decision and Information Sciences, University of Florida, Gainesville, FL, June 1992.

[12] Benson, H.P., and Morin, T.L., "A Bicriteria Mathematical Programming Model for Nutrition Planning in Developing Nations," Management Science. 33, 1593-1601 (1987). 
[13] Bitran, G.R., "Theory and Algorithms for Linear Multiple Objective Programs with Zero-One Variables," Mathematical Programming, 17, 362-390 (1979).

[14] Bitran, G.R., and Magnanti, T.L., "The Structure of Admissible Points with Respect to Cone Dominance." Journal of Optimization Theory and Applications, 29, 573-614 (1979).

[15] Cohon, J.L., Multiobjective Programming and Planning, Academic Press, New York, 1978.

[16] Dauer, J.P., "Analysis of the Objective Space in Multiple Objective Linear Programming," Journal of Mathematical Analysis and Applications, 126, 579-593 (1987).

[17] Dauer, J.P., and Liu, Y.-H., "Solving Multiple Objective Linear Programs in Objective Space," European Journal of Operational Research. 46, 350-357 (1990).

[18] Dauer, J.P., and Saleh, O.A., "Constructing the Set of Efficient Objective Values in Multiple Objective Linear Programs," European Journal of Operational Research. 46, 358-365 (1990).

[19] Dessouky, M.I., Ghiassi, M., and Davis, W.J., "Estimates of the Minimum Nondominated Criterion Values in Multiple-Criteria Decision-Making," Engineering Costs and Production Economics, 10, 95-104 (1986).

[20] Dyer, J.S., Fishburn, P.C., Steuer, R.E., Wallenius, J., and Zionts, S., "Multiple Criteria Decision Making, Multiattribute Utility Theory: The Next Ten Years," Management Science, 38, 645-654 (1992).

[21] Ecker, J.G., Hegner, N.S., and Kouada, I.A., "Generating All Maximal Efficient Faces for Multiple Objective Linear Programs," Journal of Optimization Theory and Applications, 30, 353-381 (1980).

[22] Ecker, J.G., and Kouada, I.A., "Finding all Efficient Extreme Points for Multiple Objective Linear Programs," Mathematical Programming, 14, 249-261 (1978).

[23] Evans, G.W., "An Overview of Techniques for Solving Multiobjective Mathematical Programs," Management Science, 30, 1268-1282 (1984).

[24] Evans, J.P., and Steuer, R.E., "Generating Efficient Extreme Points in Linear Multiple Objective Programming: Two Algorithms and Computing Experience," in J.L. Cochrane and M. Zeleny (Eds.), Multiple Criteria Decision Making. University of South Carolina Press, Columbia, SC, 1973, pp. 349-365.

[25] Geoffrion, A.M., "Proper Efficiency and the Theory of Vector Maximization," Journal of Mathematical Analysis and Applications, 22, 618-630 (1968).

[26] Goicoechea, A., Hansen, D.R., and Duckstein, L., Multiobjective Decision Analysis with Engineering and Business Applications, Wiley, New York, 1982.

[27] Gravel, M., Martel, J.M., Nadeau, R., Price, W., and Tremblay, R., “A Multicriterion View of Optimal Resource Allocation in Job-Shop Production," European Journal of Operational Research. 61, 230-244 (1992).

[28] Horst, R., and Pardalos, P. (Eds.), Handbook of Global Optimization. Kluwer Academic Publishers, Dordrecht, 1995.

[29] Horst, R., and Tuy, H., Global Optimization: Deterministic Approaches (2nd ed.), Springer, Berlin, 1993.

[30] Hwang, C.L., and Masud, A.S.M., "Multiple Objective Decision Making-Methods and Applications," Lecture Notes in Economics and Mathematical Systems, No. 164, Springer, Berlin, 1979.

[31] Isermann, H., "The Enumeration of the Set of All Efficient Solutions for a Linear Multiple Objective Program," Operational Research Quarterly, 28, 711-725 (1977).

[32] Keeney, R.L., and Raiffa, H., Decisions with Multiple Objectives: Preferences and Value Tradeoffs, Wiley, New York, 1976.

[33] Lee, S.M., Goal Programming for Decision Analysis. Auerbach Publishers, Philadelphia, 1972.

[34] Leschine, T.M., Wallenius, H., and Verdini, W.A., "Interactive Multiobjective Analysis and Assimilative Capacity-Based Ocean Disposal Decisions," European Journal of Operational Research, 56, 278-289 (1992).

[35] Mangasarian, O.L., Nonlinear Programming, McGraw-Hill, New York, 1969.

[36] Marcotte, O., and Soland, R.M., "An Interactive Branch-and-Bound Algorithm for Multiple Criteria Optimization," Management Science, 32, 61-75 (1986).

[37] Morse, J.N., "Reducing the Size of the Nondominated Set: Pruning by Clustering," Computers and Operations Research. 7, 55-66 (1980).

[38] Nemhauser, G.L., and Wolsey, L.A., Integer and Combinatorial Optimization, Wiley, New York, 1988. 
[39] Parker, G.R., and Rardin, R.L., Discrete Optimization, Academic Press. Boston, 1988.

[40] Prabuddha, D.. Ghosh, J.B., and Wells, C.E., "On the Minimization of Completion Time Variance with a Bicriteria Extension," Operations Research. 40, 1148-1155 (1992).

[41] Rosenthal, R.E., "Principles of Multiobjective Optimization," Decision Sciences, 16, 133- 152 (1985).

[42] Sayin, S., "Finding Discrete Representations of the Efficient Set in Multiple Objective Mathematical Programming: Theory and Methods," Ph.D. dissertation, University of Florida. Gainesville, FL. 1992.

[43] Shin, W.S., and Ravindran, A., "Interactive Multiple Objective Optimization: Survey I-Continuous Case," Computers and Operations Research, 18,97-114 (1991)

[44] Stadler, W., Ed.. Multicriteria Optimization in Enginecring and in the Sciences. Plenum, New York, 1988.

[45] Steuer, R.E., "A Five Phase Procedure for Implementing a Vector-Maximum Algorithm for Multiple Objective Linear Programming Problems," in H. Thiriez and S. Zionts (Eds.). Multiple Criteria Decision Making: Jouy-en-Josas, Springer, New York, 1975, pp. 159-168.

[46] Steuer, R.E., "Multiple Objective Linear Programming with Interval Criterion Weights," Management Science, 23, 305-316 (1976).

[47] Steuer, R.E., Muliple Criteria Optimization: Theory, Computation, and Application, Wiley. New York, 1986.

[48] Steuer. R.E., and Harris, F.W., "Intra-Set Point Generation and Filtering in Decision and Criterion Space." (omputers and Operations Research. 7, 41-53(1980).

[49] Villarreal, B., and Karwan, M.H., "Multicriteria Integer Programming: A (Hybrid) Dynamic Programming Recursive Approach," Mathematical Programming. 21, 204-223 (1981).

[50] White, D.J., "A Bibliography on the Applications of Mathematical Programming MultipleObjective Methods," Journal of the Operational Research Societ1. 41, 669-691 (1990).

[51] Yu, P.L.. Multiple-Criteria Decision Making, Plenum, New York, 1985.

[52] Yu. P.L., and Zeleny, M., "The Set of All Nondominated Solutions in Linear Cases and a Multicriteria Simplex Method," Journal of Mathematical Analysis and Applications, 49, 430$468(1975)$.

[53] Zeleny, M., "Compromise Programming," in J.L. Cochrane and M. Zeleny (Eds.), Multiplc" Criteria Decision Making. University of South Carolina Press. Columbia. SC, 1973, pp. 262301 .

[54] Zeleny, M., Multiple Criteria Decision Making, McGraw-Hill, New York, 1982.

Manuscript received December 1994

Revised manuscript received June 1996

Accepted July 9, 1996 